VoL. $61(2000) \quad[39-52]$

\title{
QUASI-BAER RING EXTENSIONS AND BIREGRULAR RINGS
}

\author{
Gary F. Birkenmeier, Jin Yong Kim and Jae Keol Park
}

\begin{abstract}
A ring $R$ with unity is called a (quasi-) Baer ring if the left annihilator of every (left ideal) nonempty subset of $R$ is generated (as a left ideal) by an idempotent. Armendariz has shown that if $R$ is a reduced PI-ring whose centre is Baer, then $R$ is Baer. We generalise his result by considering the broader question: when does the (quasi-) Baer condition extend to a ring from a subring? Also it is well known that a regular ring is Baer if and only if its lattice of principal right ideals is complete. Analogously, we prove that a biregular ring is quasi-Baer if and only if its lattice of principal ideals is complete.
\end{abstract}

Throughout $R$ will denote a ring with unity, $\mathbf{B}(R)$ its set of central idempotents, Cen $(R)$ its centre, $Z_{r}(R)$ and $Z_{\ell}(R)$ its right and left singular ideals, respectively. All subrings have a unity which may not coincide with the unity of the overring. The word "ideal" used alone (that is, without the adjectives "left" or "right") means a two-sided ideal. Recall from [19] that $R$ is a Baer ring if the right annihilator of every nonempty subset of $R$ is generated (as a right ideal) by an idempotent. The study of Baer rings has its roots in functional analysis [3] and [19]. In [19] Kaplansky introduced Baer rings to abstract various properties of von Neumann algebras and complete regular *-rings. The class of Baer rings includes the von Neumann algebras (for example, the algebra of all bounded operators on a Hilbert space), the commutative $C^{*}$-algebras $C(T)$ of continuous complex valued functions on a Stonian space $T$, and the regular rings whose lattice of principal right ideals is complete (for example, regular rings which are right continuous or right self-injective). Also the Sherman-Takeda theorem [24] and [28] shows that every $C^{*}$-algebra has a universal enveloping von Neumann algebra (hence a Baer ring).

In [12] Clark defines a ring to be quasi-Baer if the left annihilator of every ideal is generated (as a left ideal) by an idempotent (equivalently, the left annihilator of every

Received 20th March, 1999

The authors wish to thank the referee for helpful comments and suggestions. The first author gratefully acknowledges the kind hospitality he enjoyed at Busan National University and at Kyung Hee University. The second author was supported in part by Korea Research Foundation in 1997-1999, while the third author was supported in part by KOSEF, Project No. 971-0101-005-2, Korea Research Foundation Research Grant Project No. 1998-001-D00006, and the Basic Science Research Institute Program, Ministry of Education, Korea in 1998, Project No. BSRI-98-1402.

Copyright Clearance Centre, Inc. Serial-fee code: 0004-9727/00 \$A2.00+0.00. 
left ideal is generated (as a left ideal) by an idempotent). Moreover, he shows the leftright symmetry of this condition by proving that $R$ is quasi-Baer if and only if the right annihilator of every right ideal is generated (as a right ideal) by an idempotent. He then uses this condition to characterise when a finite dimensional algebra with unity over an algebraically closed field is isomorphic to a twisted matrix units semigroup algebra. Further work appeared in $[4,6,7,22]$. Every prime ring is a quasi-Baer ring. Since Baer rings are nonsingular, the prime rings $R$ with $Z_{r}(R) \neq 0[20]$ are quasi-Baer but not Baer. Every semiprime right FPF ring is quasi-Baer [14, p.168]. In [22] Pollingher and Zaks show that the class of quasi-Baer rings is closed under $n$-by- $n$ matrix rings and under $n$-by- $n$ upper (or lower) triangular matrix rings. Furthermore, it follows from their results that the quasi-Baer condition is a Morita invariant property. Thus the $n$-by- $n(n>1)$ matrix ring over a non-Prüfer commutative domain is a prime PI quasi-Baer ring which is not Baer [19, p.17]. Also the $n$-by- $n(n>1)$ upper triangular matrix ring over a domain which is not a division ring is quasi-Baer but not Baer [19, p.16]. Thus the class of quasi-Baer rings seems to behave better than the class of Baer rings under various extensions.

As motivation for this paper we recall the following results:

(1) $[1$, Theorem D] Let $R$ be a reduced (that is, $R$ has no nonzero nilpotent elements) PI-ring. If $\operatorname{Cen}(R)$ is Baer, then $R$ is a Baer ring.

(2) $[\mathbf{2 7}$, p.79] A regular ring is complete (that is, its lattice of principal right ideals is complete) if and only if it is a Baer ring.

(3) $[19$, Theorem 7] The centre of a Baer ring is a Baer ring.

As mentioned above there are prime PI rings which are not Baer. In [21, Example 3], there is a regular PI-ring $R$ with Cen $(R)$ a Baer ring, but $R$ is not Baer. However these rings are quasi-Baer.

In this paper we investigate the following questions:

(1) If the centre of a ring $R$ is Baer when is $R$ quasi-Baer?

(2) More generally, when does the quasi-Baer condition extend to a ring from a subring?

(3) Is the lattice of principal ideals of a biregular ring $R$ complete if and only if $R$ is a quasi-Baer ring?

The following results are an indication of our work:

(1) Let $S$ be a semiprime quasi-Baer subring of $R$ such that $\mathbf{B}(S) \subseteq \mathbf{B}(R)$ and every nonzero ideal of $R$ has nonzero intersection with $S$. Then $R$ is a semiprime quasi-Baer ring.

(2) Let $S$ be a semiprime quasi-Baer subring of $R$. If any of the following conditions are satisfied, then $R$ is a semiprime quasi-Baer ring: 
(i) $S$ is a right order in $R$;

(ii) $Z_{r}(S)=0$ and $R_{S}$ is an essential extension of $S_{S}$;

(iii) $R$ is the Martindale ring of quotients of $S$.

(3) Let $R$ be a semiprime PI-ring or a biregular ring. Then $R$ is a quasi-Baer ring if and only if $\operatorname{Cen}(R)$ is a Baer ring.

(4) Every separable algebra with the centre Baer is quasi-Baer. In particular, every Azumaya algebra with the centre Baer is quasi-Baer.

(5) Let $R$ be a biregular ring. Then its lattice of principal ideals is complete if and only if $R$ is a quasi-Baer ring if and only if $\operatorname{Cen}(R)$ is a Baer ring.

Recall $R$ is biregular if every principal ideal is generated by a central idempotent. Recall from [5] an idempotent $e \in R$ is left (respectively right) semicentral in $R$ if $e R e=R e$ (respectively $e R e=e R$ ). We use $\mathcal{S}_{\ell}(R)$ and $\mathcal{S}_{r}(R)$ for the sets of all left and all right semicentral idempotents of $R$, respectively. For a nonempty subset $X$ of $R, \ell_{R}(X)$ and $r_{R}(X)$ denote the left annihilator and the right annihilator of $X$, respectively. If the context is clear, we may delete the subscript $R$. If $I$ and $J$ are right (respectively left) ideals of $R$, then $I \subseteq_{r}^{e s s} J$ (respectively $I \subseteq_{\ell}^{e s s} J$ ) denotes $I$ is essential in $J$ as $R$-modules.

\section{Preliminaries and Examples}

In this section we establish some elementary properties of quasi-Baer rings which are necessary for our main results in the following sections.

LEMMA 1.1. For an idempotent $e$ of $R$, the following conditions are equivalent:

(i) $e \in \mathcal{S}_{\ell}(R)$;

(ii) $1-e \in \mathcal{S}_{r}(R)$;

(iii) $x e=e x e$, for each $x \in R$;

(iv) $(1-e) R e=0$;

(v) $(1-e) x=(1-e) x(1-e)$, for each $x \in R$;

(vi) $e R$ is an ideal of $R$;

(vii) $R(1-e)$ is an ideal of $R$;

(viii) $e R(1-e)$ is an ideal of $R$ and $e R=e R(1-e) \oplus R e$, as a direct sum of left ideals.

Proposition 1.2. A ring $R$ is quasi-Baer if and only if whenever $I$ is an ideal of $R$ there exists $e \in \mathcal{S}_{\ell}(R)$ such that $I \subseteq e R$ and $\ell(I) \cap e R=e R(1-e)$.

Proof: Assume that $R$ is quasi-Baer and $I$ is an ideal of $R$. Then there exists $c \in \mathcal{S}_{r}(R)$ such that $\ell(I)=R c$. Then $I \subseteq(1-c) R$. Let $e=1-c$. By Lemma 1.1, $e \in \mathcal{S}_{\ell}(R)$. Now $\ell(I) \cap e R=e R \cap R(1-e)=e R(1-e)$. 
Conversely, let $I$ be an ideal of $R$, and assume there exsits $e \in \mathcal{S}_{\ell}(R)$ such that $l \subseteq e R$ and $\ell(I) \cap e R=e R(1-e)$. Let $\alpha \in \ell(I)$. Then $\alpha=\alpha e+\alpha(1-e)$. So $e \alpha=e \alpha e+e \alpha(1-e)$. But $e \alpha \in \ell(I) \cap e R$. Hence $e \alpha=e \alpha(1-e)$, so $e \alpha e=0$. But $e \in \mathcal{S}_{\ell}(R)$, so $0=$ eae $=\alpha e$ by Lemma 1.1. Thus $\alpha=\alpha(1-e) \in R(1-e)$. Hence $\ell(I)=R(1-e)$. Therefore $R$ is a quasi-Baer ring.

Observe that if $R$ is quasi-Baer and $I$ is an ideal of $R$, the idempotent $e$ in Proposition 1.2 satisfies $e R=r(\ell(I))$.

Corollary 1.3. If $R$ is a quasi-Baer ring and $I$ is an ideal of $R$, then there exsits $e \in \mathcal{S}_{\ell}(R)$ such that $I \subseteq e R$ and $I+e R(1-e)$ is right essential in $e R$ and $e R(1-e)$ is an ideal of $R$. In particular, if $I$ contains the prime radical of $R$ (for example, $R$ is semiprime) or $e$ is central, then $I$ is right essential in $e R$. Moreover if $I$ is not right essential in $e R$, then there exists a closed right ideal $0 \neq X=e X(1-e)$ such that $I \cap X=0$ and $I \oplus X \subseteq_{r}^{e s s} e R$.

From Corollary 1.3, a right duo quasi-Baer (hence Baer) ring is a right CS-ring. However there are commutative self-injective rings which are not Baer (for example, $\mathbb{Z}_{4}$ ). Thus Corollary 1.3 has no converse.

PROPOSITION 1.4. Let $R$ be a quasi-Baer ring.

(i) $R$ is semiprime if and only if $\mathcal{S}_{\ell}(R)=\mathrm{B}(R)$.

(ii) If every essential right ideal of $R$ is an essential extension of an ideal of $R$ (for example, if $R$ has essential right socle), then $R$ is right nonsingular.

Proof: (i) For any $e \in \mathcal{S}_{\ell}(R)$, by Lemma 1.1, $e R(1-e)$ is an ideal of $R$ and $(1-e) R e=0$. Note that $e R(1-e)$ is nilpotent. If $R$ is semiprime, $e R(1-e)=0$ and so $e \in \mathbf{B}(R)$. Hence $\mathcal{S}_{\ell}(R)=\mathbf{B}(R)$. Conversely, assume $\mathcal{S}_{\ell}(R)=\mathbf{B}(R)$. Suppose that there exists a nonzero ideal $I$ of $R$ such that $I^{2}=0$. Then there exists $0 \neq e \in \mathbf{B}(R)$ such that $r(I)=e R$. But $I \subseteq r(I)$. So $I=e I=I e=0$, a contradiction. Therefore $R$ is semiprime.

(ii) Let $0 \neq x \in Z_{r}(R)$. Then $r(x) \subseteq_{r}^{e s s} R$. Hence there exists an ideal $I$ of $R$ such that $I \subseteq r(x)$ and $I \subseteq_{r}^{e s s} R$. So $x I=0$. Thus $I \subseteq r(x R)$ and $r(x R)=e R$ for some $e=e^{2} \in R$. Since $I$ is right essential in $R$. Then $e=1$. So $x=0$, a contradiction.

The next two results give conditions which guarantee when the quasi-Baer condition implies the Baer condition. $R$ is called an Abelian ring if every idempotent of $R$ is central.

Proposition 1.5. The following conditions are equivalent:

(i) $R$ is an Abelian Baer ring.

(ii) $R$ is a reduced quasi-Baer ring. 
Proof: (i) $\Rightarrow$ (ii). Let $0 \neq x \in R$ such that $x^{2}=0$. Then there exists $e \in \mathbf{B}(R)$ such that $r(x)=e R$. Then $x=e x=x e=0$. Hence $R$ is reduced. Clearly $R$ is quasi-Baer.

(ii) $\Rightarrow$ (i). This part is a consequence of $[4$, Lemma 1$]$.

Recall from [2] that $R$ is said to satisfy the IFP (insertion of factors property) if $r_{R}(x)$ is an ideal of $R$ for all $x \in R$ (equivalently, $r_{R}(X)$ is an ideal of $R$ for all nonempty subsets $X$ of $R$ ). $R$ is strongly right (resptively left) bounded if every nonzero right (respectively left) ideal contains a nonzero ideal. $R$ is strongly bounded if it is both strongly right and strongly left bounded.

Proposition 1.6. Let $R$ be a quasi-Baer ring. If $R$ satisfies either of the following conditions, then $R$ is a reduced Baer ring.

(i) $R$ has IFP.

(ii) $R$ is strongly right bounded.

Proof: If $R$ has IFP, then it is immediate that $R$ is a Baer ring. By [25, Lemma 2.7], $R$ is Abelian. From Proposition 1.5, $R$ is reduced. Now assume that $R$ is strongly right bounded. Let $\emptyset \neq X \subseteq R$. Then $r(R X R)=e R \subseteq r(X)$ for some idempotent $e \in R$. Hence $r(X)=e R \oplus A$ for some $A$. If $A \neq 0$, there exists a nonzero ideal $B \subseteq A$. Then $(R X R) B=0$. So $B \subseteq e R$, a contradiction. Therefore $R$ is a Baer ring. Suppose $x \neq 0$ but $x^{2}=0$. Then there exists a nonzero ideal $I$ of $R$ such that $I \subseteq x R$ and $I^{2}=0$. Then $r(I)=f R$ for some idempotent $f$. Then $I \subseteq f R$. Also there exists a nonzero ideal $J$ of $R$ such that $J \subseteq(1-e) R$. Then $J \subseteq r(I)$, a contradiction. Therefore $R$ is reduced.

A ring $R$ is said to be right $P P$ if every principal right ideal of $R$ is projective. Note that a ring $R$ is right $P P$ if and only if for any $x \in R$ there is an idempotent $e$ in $R$ such that $r(x)=e R$. Similarly left $P P$ ring can be defined. A ring $R$ is called $P P$ if it is both right and left PP. Since a left hereditary ring is left PP and if it has no infinite set of pairwise orthogonal idempotents, then it is Baer [26], so one may raise the following question: If $R$ is a left hereditary ring, is it quasi-Baer? The following example, due to Chase [9] (see also [10, Example 8.2]), answers this question in the negative.

EXAMPLE 1.7. In $A=\prod_{n=1}^{\infty} \mathbf{Z}_{2}$, consider $T=\left\{\left(a_{n}\right)_{n=1}^{\infty} \mid a_{n}\right.$ is eventually constant $\}$ and $I=\left\{\left(a_{n}\right)_{n=1}^{\infty} \mid a_{n}=0\right.$ eventually $\}=\bigoplus_{n=1}^{\infty} \mathbb{Z}_{2}$. Now let $R=\left(\begin{array}{cc}T / I & T / I \\ 0 & T\end{array}\right)$. Then by Chase [9], the ring $R$ is a left hereditary ring. Let

$$
\alpha=\left(\begin{array}{cc}
(0,0, \ldots)+I & (1,1, \ldots)+I \\
0 & (0,0, \ldots)
\end{array}\right) \in R
$$


Then $\alpha R=\left(\begin{array}{cc}0 & T / I \\ 0 & 0\end{array}\right)$. In this case $r(\alpha R)=\left(\begin{array}{cc}T / I & T / I \\ 0 & I\end{array}\right)$. But there does not exist an idempotent $e \in R$ such that $r(\alpha R)=e R$.

PROPOSITION 1.8. The centre of a quasi-Baer ring is a Baer ring.

Proof: Let $R$ be a quasi-Baer ring. Then for a nonempty subset $X$ of $\operatorname{Cen}(R)$ there exists $e \in \mathcal{S}_{\ell}(R)$ such that $r_{R}(X)=r_{R}(X R)=e R$. But $r_{R}(X)=\ell_{R}(X)=$ $R f$ for some idempotent $f$. Since $e R=R f$, it follows that $e=f \in \operatorname{Cen}(R)$. So $r_{\text {Cen }(R)}(X)=e \operatorname{Cen}(R)$. Therefore $\operatorname{Cen}(R)$ is a Baer ring.

In general the converse of Proposition 1.8 does not hold.

EXAMPLe 1.9. For a field $F$, let

$$
R=\left(\begin{array}{ccc}
F & \operatorname{Mat}_{2}(F) & \operatorname{Mat}_{2}(F) \\
0 & F & \operatorname{Mat}_{2}(F) \\
0 & 0 & F
\end{array}\right),
$$

which is a subring of the 3-by-3 upper triangular matrix ring over $\operatorname{Mat}_{2}(F)$. Then $R$ is a PI-ring whose centre is Baer, but the ring $R$ is not quasi-Baer.

In the next section we determine conditions for which the converse of Proposition 1.8 does hold.

\section{Extending THE QUASI-BAER CONDITION}

In this section first we consider the problem of extending the quasi-Baer condition to a ring from one of its subrings with semiprime condition. Then we also consider extending the quasi-Baer ring condition from subrings without semiprime condition, but with strengthening the intersection condition. We use our results to generalise a result of Armendariz [1].

LEMMA 2.1. Let $S$ be a semiprime quasi-Baer subring of $R$ with unity $1_{S}$ such that $\mathrm{B}(S) \subseteq \mathcal{S}_{\ell}(R)$ and for each nonzero ideal $I$ of $R$ if $r_{R}(I) \neq 0$ then $r_{S}(I \cap S) \subseteq$ $r_{R}(I)$ and $I \cap S \neq 0$. Then $R$ is a semiprime quasi-Baer ring and $1_{S}=1_{R}$.

Proof: Suppose $K$ is a nonzero ideal of $R$ such that $K^{2}=0$. Then $(K \cap S)^{2}=0$ and $K \cap S \neq 0$, a contradiction. Therefore $R$ is a semiprime ring and so $\mathrm{B}(S) \subseteq$ $\mathcal{S}_{\ell}(R)=\mathbf{B}(R)$ by assumption and Proposition 1.4. Thus $1_{S} \in \mathbf{B}(R)$. Since $\left[\left(1_{S}-1_{R}\right) R\right] \cap S=0$, it follows that $\left(1_{S}-1_{R}\right) R=0$. So $1_{S}=1_{R}$.

Now assume $I$ is a nonzero ideal of $R$ such that $r_{R}(I) \neq 0$. Then $r_{S}(I \cap S)=e S$, for some $e \in \mathbf{B}(S)$, and $I \cap S \neq 0$. Hence $r_{R}(I)=e R \oplus A$ for some ideal $A$ of $R$.

First assume $e=0$ and consider the following cases:

CASE 1. Assume $r_{R}(A) \neq 0$. Since $A$ is a nonzero ideal of $R, r_{S}(A \cap S) \subseteq r_{R}(A)$ and $A \cap S \neq 0$. Let $0 \neq s \in A \cap S$. Then $s \in r_{S}(I \cap S)=0$, a contradiction. 
CAse 2. Assume $r_{R}(A)=0$. Then $A I \neq 0$. So $0 \neq A \subseteq r_{R}(A I)$. Hence there exists $0 \neq s \in A I \cap S$. But $s \in r_{S}(I \cap S)=0$, a contradiction.

Consequently, it follows that $e \neq 0$. If $A=0$, we are finished. So assume $A \neq 0$. Since $0 \neq e R \subseteq r_{R}(A)$, there exists $0 \neq s \in A \cap S$. Then $s \in r_{S}(I \cap S) \subseteq e R$, a contradiction. Hence $A=0$ and so $r_{R}(I)=e R$. Therefore $R$ is quasi-Baer.

THEOREM 2.2. Let $S$ be a semiprime quasi-Baer subring of $R$ with unity $1_{S}$ such that $\mathrm{B}(S) \subseteq \mathcal{S}_{\mathcal{l}}(R)$ and every nonzero ideal of $R$ has nonzero intersection with $S$. Then $R$ is a semiprime quasi-Baer ring and $1_{S}=1_{R}$.

Proof: As in the proof of Lemma 2.1, $R$ is semiprime and $1_{S}=1_{R}$. Assume that $I$ is a nonzero ideal of $R$. If $r_{R}(I)=0$, then $r_{R}(I)$ is generated by an idempotent. So assume $r_{R}(I) \neq 0$. Then $r_{S}(I \cap S)=e S$ where $e \in \mathbf{B}(S) \subseteq \mathcal{S}_{\ell}(R)=\mathbf{B}(R)$ and $I \cap S \neq 0$. Suppose $I e \neq 0$. Then there exsits $0 \neq s \in I e \cap S$. So $0 \neq(I \cap S) \cap r_{S}(I \cap S)$, a contradiction to the semiprimeness of $S$. Thus $I e=0$, so $r_{S}(I \cap S) \subseteq r_{R}(I)$. By Lemma 2.1, $R$ is a quasi-Baer ring.

Corollary 2.3. Let $S$ be a semiprime quasi-Baer subring of a ring $R$. If any of the following conditions are satisfied, then $R$ is a semiprime quasi-Baer ring.

(i) $R \subseteq Q_{r}(S)$, where $Q_{r}(S)$ is the maximal ring of right quotients of $S$.

(ii) $S$ is a right order in $R$.

(iii) $R$ is the Martindale ring of quotients of $S$.

Proof: For the conditions (i)-(iii), $R_{S}$ is an essential extension of $S_{S}$ and $\mathbf{B}(S) \subseteq$ $\mathrm{B}(R)$. Thus the result is a consequence of Theorem 2.2.

THEOREM 2.4. Let $S$ be a quasi-Baer subring of a ring $R$ such that every nonzero ideal of $R$ has nonzero intersection with $S$. If $R$ satisfies any of the following conditions, then $R$ is a semiprime right nonsingular quasi-Baer ring and $S$ is a reduced Baer ring.

(i) For each $s \in S, r_{R}(s)$ is an ideal of $R$.

(ii) $R$ has the IFP.

(iii) $S$ is contained in the centre of $R$.

Proof: Assume condition (i). Then $S$ has the IFP. By Propositions 1.5 and 1.6, $S$ is a reduced Baer ring. As in Lemma 2.1, $1_{S}=1_{R}$. Let $e \in \mathbf{B}(S)$. Then $1_{R}-e \in S$. So $r_{R}\left(1_{R}-e\right)=e R$ is an ideal of $R$. Hence $e \in \mathcal{S}_{\ell}(R)$. By Theorem 2.2, $R$ is a semiprime quasi-Baer ring. Assume $Z_{r}(R) \neq 0$. Then there exists $0 \neq s \in S \cap Z_{r}(R)$. Hence $r_{S}(s)=r_{R}(s R)=c R$ for some $c \in \mathbf{B}(R)$, a contradiction. Therefore $R$ is right nonsingular. Observe that conditions (ii) and (iii) both imply condition (i). Also note that for conditions (ii) and (iii), $R$ is also left nonsingular.

Corollary 2.5. Assume that $R$ is a biregular ring or a semiprime PI-ring. 
Then $R$ is a quasi-Baer ring if and only if $\operatorname{Cen}(R)$ is a Baer ring.

Proof: Assume that Cen $(R)$ is a Baer ring. By definition, a biregular ring satisfies the condition that every nonzero ideal has nonzero intersection with the centre. This is also true for semiprime PI-rings [23]. The result is now a consequence of Theorem 2.4 (iii). The converse follows from Proposition 1.8.

EXAMPLE 2.6.

(i) There is a biregular ring $R$ with the centre Baer, but $R$ is not Baer (see Example 3.6).

(ii) Let $R$ be a non-Prüfer commutative domain. Then by [26], there is a positive integer $n$ such that $\operatorname{Mat}_{n}(R)$ is not Baer, but it is semiprime PI with the centre Baer.

(iii) There is a semiprime ring which is neither PI nor biregular, but every nonzero ideal has nonzero intersection with the centre and the centre is a Baer ring (see Theorem 2.4 (iii)). Let $R=\mathbb{Z}\{x, y\} /(y x-x y-1\rangle$, where $\mathbb{Z}\{x, y\}$ is the free algebra over the ring $\mathbb{Z}$ of integers and $\langle y x-x y-1\rangle$ is the ideal of $\mathbb{Z}\{x, y\}$ generated by $y x-x y-1$. Then $R$ is a domain which is neither PI nor biregular. But every nonzero ideal contains a nonzero central element. Also the centre of $R$ is $\mathbb{Z}$.

The condition that $R$ is a PI-ring in Corollary 2.5 is not superfluous. Indeed, by the second example in [1, p.471] and [21, Example 5], there are reduced rings $R$ with the centre Baer, but $R$ is not quasi-Baer. Moreover, the condition that $R$ is semiprime in Corollary 2.5 is not superfluous. Let $R$ be the ring in Example 1.9. Then $R$ is a PI-ring with the centre Baer. But $R$ is not quasi-Baer.

Note that semiprime PI-rings are nonsingular rings by [15] and have bounded index of nilpotency by [18, Theorem 10.8.2]. So one may raise the following question from Corollary 2.5: Assume that $R$ is a nonsingular PI-ring of bounded index of nilpotency. If the centre is Baer, then is $R$ quasi-Baer? But the ring $R$ in Example 1.9 is a nonsingular PI-ring of bounded index of nilpotency and the centre is Baer. But $R$ is not quasi-Baer.

For any $C$-algebra $R$ ( $C$ is a commutative ring with unity), we can form the so called enveloping algebra $R^{e}=R \bigotimes_{C} R^{\circ}$, where $R^{o}$ denotes the $C$-algebra opposite to $R$. The algebra $R$ has a structure as a left $R^{e}$-module induced by $(x \otimes y) \cdot r=x r y$. Recall that a $C$-algebra $R$ is called separable if $R$ is a projective left $R^{e}$-module. A separable algebra $R$ is called an Azumaya algebra if $C$ is the centre of $R$, that is, $R$ is a central separable algebra.

Corollary 2.7. Every separable algebra with the centre Baer is quasi-Baer. In particular, every Azumaya algebra with the centre Baer is quasi-Baer. 
Proof: Let $R$ be a separable algebra. Then by [13, Theorem 3.8] $R$ is separable as an algebra over its centre. By [13, Corollary 3.7, p.54], every nonzero ideal of $R$ has nonzero intersection with the centre. Thus by Theorem 2.4 (iii), $R$ is quasi-Baer.

From now on we consider extending the quasi-Baer ring condition from subrings without semiprime condition. But we strengthen the intersection condition.

THEOREM 2.8. Let $S$ be a quasi-Baer subring of a ring $R$ such that every nonzero one-sided ideal of $R$ has nonzero intersection with $S$. If $R$ is left (or right) nonsingular, then $R$ is quasi-Baer.

Proof: Assume that $R$ is left nonsingular. Let $I$ be a nonzero ideal of $R$. Then there exists $e=e^{2} \in S$ such that $r_{S}(I \cap S)=e S$. Observe $R(I \cap S) \subseteq_{\ell}^{e s s} I$. Since $R$ is left nonsingular, $r_{R}(R(I \cap S))=r_{R}(I)$. Hence $r_{R}(I)=e R \oplus A$ for some right ideal $A$ of $R$. If $A \neq 0$, then there exists $0 \neq s \in A \cap S$. Then $s \in r_{S}(I \cap S) \subseteq e R$, a contradiction. Therefore $r_{R}(I)=e R$. Consequently, $R$ is a quasi-Baer ring. The argument is similar when $R$ is right nonsingular.

COROLlaRY 2.9. Let $S$ be a nonsingular quasi-Baer ring whose maximal right and left quotient rings coincide. If $S$ is a subring of $R$ such that $R_{S}$ (or ${ }_{S} R$ ) is an essential extension of $S$, then $R$ is quasi-Baer.

Proof: By [16, Exercise 12, p.68], if $R_{S}$ is an essential extension of $S_{S}$, then $R$ is both a right and a left quotient ring of $S$. From [16, Proposition 2.32], $R$ is nonsingular. Now Theorem 2.8 yields the result.

Note if $R$ is a right nonsingular CS-ring, then $R$ is a Baer ring [11] whose maximal right and left quotient rings of $R$ coincide [29]. For our next result, note that Baer rings, right hereditary rings and regular rings are right $P P$ rings.

Corollary 2.10. Let $S$ be a right PP quasi-Baer subring of $R$ such that every one-sided ideal of $R$ has nonzero intersection with $S$. Then $R$ is a nonsingular quasi-Baer ring.

Proof: Assume that $Z_{r}(R) \neq 0$. Then there exists $0 \neq s \in Z_{r}(R) \cap S$. So $r_{S}(s)=e S$ for some $0 \neq e=e^{2} \in S$. Thus $r_{R}(s)=e R \oplus A$ for some right ideal $A$ of $R$. If $A \neq 0$, then there exists $0 \neq a \in A \cap S$. So $a \in r_{S}(s) \subseteq e R$, a contradiction. Similarly, $Z_{\ell}(R)=0$. The result is now a consequence of Theorem 2.8 .

Observe that [21, Example 3] and Example 3.7 are regular (hence PP) quasi-Baer rings which are not Baer.

COROLLARY 2.11. Let $S$ be a Baer subring of $R$ such that every nonzero ideal of $R$ has nonzero intersection with $S$. If $R$ is a strongly bounded ring, then $R$ is a reduced Baer ring.

Proof: Since $R$ is strongly bounded, every nonzero one-sided ideal has nonzero 
intersection with $S$. Since $S$ is a Baer ring, it is both a right and a left PP ring. Now the result follows from Corollary 2.10 and Proposition 1.6.

Now we show that Corollaries 2.5 and 2.11 are generalisations of Armendariz's result $[1$, Theorem $\mathrm{D}]$.

Corollary 2.12. [1, Theorem D] Let $R$ be a reduced PI-ring. If the centre of $R$ is a Baer ring, then $R$ is a Baer ring.

Proof: This result is a consequence of Corollary 2.5 and Proposition 1.5. Alternatively, from [1, Theorem $\mathrm{C}]$ we have that $R$ is strongly bounded. By [23] every nonzero ideal has nonzero intersection with the centre. The result is now a consequence of Corollary 2.11 .

\section{Biregular Rings}

Observe that biregular rings are "almost" quasi-Baer in the sense that the right annihilator of every principal ideal is generated by an idempotent. Also every regular ring is PP (which is "almost" Baer). It is well known [27, p.79] that a regular ring is Baer if and only if its lattice of principal right ideals is complete. These observations motivate us to ask: Can the quasi-Baer condition on a biregular ring be characterised by the completeness of the lattice of its principal ideals?

In this section we answer this question in the affirmative and apply some of the results of previous sections. Our results rely on the methods used in [27] for analogous results. We first show that, in general, a biregular ring is not quasi-Baer and vice versa.

EXAMPLE 3.1.

(i) Let $W$ be the first Weyl algebra over a field of characteristic zero and let $R=\left\{\left(x_{n}\right) \in \prod_{n=1}^{\infty} W \mid x_{n}\right.$ is eventually constant $\}$. Then it can be easily checked that the ring $R$ is biregular, but not quasi-Baer.

(ii) For a positive integer $n>1$, the $n$-by- $n$ upper triangular matrix ring over a field is quasi-Baer, but not biregular.

The following lemma was proved in [12, Theorem 2] for the case of Artinian quasiBaer rings.

LEMMA 3.2. The set of right ideals generated by left semicentral idempotents of $R$ is a distributive sublattice of the lattice of ideals of $R$. More generally, if $\left\{e_{\lambda}\right\}_{\lambda \in \Lambda} \subseteq$ $\mathcal{S}_{\ell}(R)$ and $c \in \mathcal{S}_{\ell}(R)$, then $\left(\sum_{\Lambda} e_{\lambda} R\right) \cap c R=\sum_{\Lambda}\left(e_{\lambda} R \cap c R\right)$.

Proof: Let $c, e_{1}, e_{2} \in \mathcal{S}_{\ell}(R)$. Hence $e_{1} R$ and $e_{2} R$ are ideals of $R$ by Lemma 1.1. Let $e=e_{1}+e_{2}-e_{1} e_{2}$. By direct calculation we have: $e \in \mathcal{S}_{\ell}(R) ; e_{1} R+e_{2} R=e R$; $e_{1} R \cap e_{2} R=e_{1} e_{2} R$; and $e_{1} e_{2} \in \mathcal{S}_{\ell}(R)$. Also we have $\left(e_{1} R+e_{2} R\right) \cap c R=e R \cap c R=e c R$, 
and $\left(e_{1} R \cap c R\right)+\left(e_{2} R \cap c R\right)=e_{1} c R+e_{2} c R=\left(e_{1} c+e_{2} c-e_{1} c e_{2} c\right) R=e c R$. The general distributive law follows routinely from the case $\Lambda=\{1,2\}$. Therefore the set of right ideals generated by the left semicentral idempotents is a distributive sublattice of the lattice of ideals of $R$.

Lemma 3.3. Let $R$ be a quasi-Baer ring. Then the set of principal right ideals generated by the left semicentral idempotents of $R$ is a complete sublattice of the lattice of ideals of $R$.

Proof: Let $\left\{e_{\lambda}\right\}_{\lambda \in \Lambda} \subseteq \mathcal{S}_{\ell}(R)$. Observe that $R\left(1-e_{\lambda}\right)$ is an ideal of $R$ for each $\lambda \in \Lambda$ by Lemma 1.1. Then there exists an idempotent $e$ such that $\bigcap_{\lambda \in \Lambda} e_{\lambda} R=$ $\bigcap_{\lambda \in \Lambda} r\left(R\left(1-e_{\lambda}\right)\right)=r\left(\sum_{\Lambda} R\left(1-e_{\lambda}\right)\right)=e R$. Hence every subset of principal right ideals generated by left semicentral idempotents has a greatest lower bound. So the lattice of principal right ideals generated by the left semicentral idempotents is complete.

The following lemma is probably well known but we include a proof for completeness.

LеммA 3.4. The centre of a biregular ring is regular.

Proof: Let $x \in \operatorname{Cen}(R)$. Then there exists $e \in \mathrm{B}(R)$ such that $x R=e R$. Since $x=e x$, we have $x \operatorname{Cen}(R) \subseteq e \operatorname{Cen}(R)$. Also there exists $r \in R$ such that $e=x r=x e r=r x$. Let $y \in R$. Then $(e r) y-y(e r)=e(r y-y r)=r x(r y-y r)=$ $r(x r y-x y r)=r(x r y-y x r)=r(e y-y e)=r e(y-y)=0$. Thus $e r \in \operatorname{Cen}(R)$. So $e \in x \operatorname{Cen}(R)$. Hence $x \operatorname{Cen}(R)=e \operatorname{Cen}(R)$.

THEOREM 3.5. Let $R$ be a biregular ring. Then the following conditions are equivalent:

(i) $R$ is a quasi-Baer ring.

(ii) The lattice of principal ideals of $R$ is complete.

(iii) The Boolean ring of central idempotents of $R$ is a self-injective ring.

(iv) The centre of $R$ is a Baer ring.

(v) The centre of $R$ is a continuous ring.

Proof: (i) $\Rightarrow$ (ii) Since $R$ is semiprime, $\mathcal{S}_{\ell}(R)=\mathbf{B}(R)$ by Proposition 1.4. Thus every principal ideal is the right ideal generated by some left semicentral idempotent. By Lemma 3.2, the set of principal ideals forms a sublattice of the lattice of ideals of $R$. Hence this implication is a consequence of Lemma 3.3.

(ii) $\Rightarrow$ (iii) Let $\left\{e_{\lambda}\right\}_{\lambda \in \Lambda} \subseteq \mathbf{B}(R)$. Then there exists $e \in \mathbf{B}(R)$ such that $\bigcap_{\Lambda} e_{\lambda} R=$ $e R$. It follows that $\bigcap_{\Lambda} e_{\lambda} \mathbf{B}(R)=e \mathbf{B}(R)$. Hence the Boolean ring of central idempotents of $R$ is complete. By [27, p.250], the Boolean ring of central idempotents of $R$ is selfinjective. 
(iii) $\Rightarrow$ (ii) Let $\left\{e_{\lambda}\right\}_{\lambda \in \Lambda} \subseteq \mathbf{B}(R)$. By [27, pp.249-250], there exists $e \in \mathbf{B}(R)$ such that $\bigcap_{\Lambda} e_{\lambda} \mathrm{B}(R)=e \mathrm{~B}(R)$. Clearly $e R \subseteq \bigcap_{\Lambda} e_{\lambda} R$. Let $x \in \bigcap_{\Lambda} e_{\lambda} R$. There exists $c \in \mathrm{B}(R)$ such that $R x R=c R$. Then $e_{\lambda} c=c$, for all $\lambda \in \Lambda$. So $c \in e \mathbf{B}(R)$. Hence $R x R \subseteq e R$. Thus $\bigcap e_{\lambda} R=e R$. Also we may check easily that $f R$ is the greatest lower bound of $\left\{e_{\lambda} R \mid \lambda \in \Lambda\right\}$ as in Lemma 3.3. So the lattice of principal ideals of $R$ is complete.

(ii) $\Rightarrow$ (i) Let $I$ be an ideal of $R$. There exists $c \in \mathbf{B}(R)$ such that $c R$ is the greatest lower bound of the principal ideals $(1-e) R$, for all central idempotents $e$ in $\ell(r(I))$. Let $J=\bigcap_{e \in \ell(r(I))}(1-e) R$. Then $c R \subseteq J$. Hence $J=c R \oplus W$. Let $w \in W$. There exists $v \in \mathbf{B}(R)$ such that $R w R=v R$. Then $c R \oplus v R=(c+v) R$, where $c+v \in \mathbf{B}(R)$. Since $c R$ is the greatest lower bound, then $c R \subseteq(c+v) R \subseteq c R$. So $W=0$, and $c R=J$. Let $b \in \ell(r(I))$. Then there exists $d \in \mathbf{B}(R)$ such that $R b R=d R$. Hence $d \in \ell(r(I))$. So $c R \subseteq(1-d) R$. Thus $c R \subseteq r(b)$. Hence $c R \subseteq r(\ell(r(I)))=r(I)$. Now let $a \in r(I)$. Then $a=(1-e) a \in(1-e) R$, for every central idempotent $e \in \ell(r(I))$. So $a \in c R$. Therefore $r(I)=c R$.

(i) $\Leftrightarrow$ (iv) This equivalence is a direct consequence of Proposition 1.8 and Corollary 2.5 .

(iv) $\Rightarrow$ (v) By Lemma 3.4, the centre of $R$ is a commutative regular ring. Hence the centre is cononsingular. By [11, Theorem 2.1] and [19, Theoem 7], the centre is a CS regular ring. From [17, Corollary 13.4], the centre is a continuous ring.

(v) $\Rightarrow$ (i) Since the centre is a continuous regular ring, then it is a nonsingular CS ring. By [11, Theorem 2.1], the centre is a Baer ring. Now Corollary 2.5 yields that $R$ is a quasi-Baer ring.

EXAMPLE 3.6. There are rings $R$ such that

(1) $R$ is biregular quasi-Baer, but

(2) $R$ is neither regular nor Baer.

(i) For a positive integer $k>1$, let $W$ be the $k$-th Weyl algebra over a field of chracteristic zero. Then $W$ is a simple Noetherian domain which is neither left nor right hereditary. So there exists a positive integer $n$ such that $\operatorname{Mat}_{n}(W)$ is neither left nor right PP by [26]. Hence it is not Baer. Obviously it is not regular.

(ii) By Zalesskii and Neroslavskii [10, Example 14.17, p.179], there is a simple Noetherian ring $R$ which is not a domain and in which 0 and 1 are the only idempotents. Then $R$ is an Abelian quasi-Baer ring which is neither regular nor Baer.

EXAMPLE 3.7. [8, Example 1.17] There is a ring $R$ such that

(1) $R$ is regular quasi-Baer, but

(2) $R$ is neither biregular nor Baer. 
For a finite field $F$, let

$$
R=\left\{\left(\begin{array}{cccc}
A & & & 0 \\
& a & & \\
& & a & \\
0 & & & \ddots
\end{array}\right) \mid A \in \operatorname{Mat}_{n}(F), a \in F, n=1,2, \ldots\right\} .
$$

Then $R$ is prime regular, so $R$ is quasi-Baer. But $\mathrm{R}$ cannot be Baer because of [8, Theorem 1.14]. Also it can be easily checked that $R$ cannot be biregular.

\section{REFERENCES}

[1] E.P. Armendariz, 'A note on extensions of Baer and p.p.-rings', J. Austral. Math. Soc. 18 (1974), 470-473.

[2] H.E. Bell, 'Near-rings in which each element is a power of itself', Bull. Austral. Math. Soc. 2 (1970), 363-368.

[3] S.K. Berberian, Baer *-Rings (Springer-Verlag, Berlin, Heidelberg, New York, 1972).

[4] G.F. Birkenmeier, 'Baer rings and quasi-continuous rings have a MDSN', Pacific J. Math. 97 (1981), 283-292.

[5] G.F. Birkenmeier, 'Idempotents and completely semiprime ideals', Comm. Algebra 11 (1983), 567-580.

[6] G.F. Birkenmeier, 'Decompositions of Baer-like rings', Acta Math. Hungar. 59 (1992), 319-326.

[7] G.F. Birkenmeier, H.E. Heatherly, J.Y. Kim and J.K. Park, 'Triangular matrix representations', (preprint).

[8] G.F. Birkenmeier, J.Y. Kim and J.K. Park, 'Rings with countably many direct summands', Comm. Algebra (to appear).

[9] S. Chase, 'A generalization of the ring of triangular matrices', Nagoya Math. J. 18 (1961), 13-25.

[10] A.W. Chatters and C.R. Hajarnavis, Rings with chain conditions (Pitman, Boston, 1980).

[11] A.W. Chatters and S.M. Khuri, 'Endomorphism rings of modules over nonsingular CS-rings', J. London Math. Soc. (2) 21 (1980), 434-444.

[12] W.E. Clark, 'Twisted matrix units semigroup algebras', Duke Math. J. 34 (1967), 417-424.

[13] F. DeMeyer and E. Ingraham, Separable algebras over commutative rings, Lecture Notes in Math. 181 (Springer-Verlag, Berlin, Heidelberg, New York, 1971).

[14] C. Faith, 'Injective quotient rings of commutative rings', in Module theory, Springer Lecture Notes 700 (Springer-Verlag, Berlin, Heidelberg, New York, 1979), pp. 151-203.

[15] J.W. Fisher, 'Structure of semiprime P.I. rings', Proc. Amer. Math. Soc. 39 (1973), 465-467.

[16] K.R. Goodearl, Ring theory - nonsingular rings and modules (Marcel Dekker, New York, 1976). 
[17] K.R. Goodearl, Von Neumann regular rings (Pitman, London, 1979).

[18] N. Jacobson, Structure of rings, Amer. Math. Soc. Colloq. Publ. 37 (American Mathematical Society, Providence, 1964).

[19] I. Kaplansky, Rings of operators (Benjamin, New York, 1965).

[20] J. Lawrence, 'A singular primitive ring', Proc. Amer. Math. Soc. 45 (1974), 59-62.

[21] Y. Lee, N.K. Kim and C.Y. Hong, 'Counterexamples in Baer rings', Comm. Algebra 25 (1997), 497-507.

[22] A. Pollingher and A. Zaks, 'On Baer and quasi-Baer rings', Duke Math. J. 37 (1970), 127-138.

[23] L. Rowen, 'Some results on the center of a ring with polynomial identity', Bull. Amer. Math. Soc. 79 (1973), 219-223.

[24] S. Sherman, 'The second adjoint of a $C^{*}$-algebra', Proc. Intern. Cong. Math. Cambridge $1(1950), 470$.

[25] G. Shin, 'Prime ideals and sheaf representation of a pseudo symmetric ring', Trans. Amer. Math. Soc. 184 (1973), 43-61.

[26] L.W. Small, 'Semihereditary rings', Bull. Amer. Math. Soc. 73 (1967), 656-658.

[27] B. Stenström, Rings of quotients (Springer-Verlag, Berlin, Heidelberg, New York, 1975).

[28] Z. Takeda, 'Conjugate spaces of operator algebras', Proc. Japan Acad. 30 (1954), 90-95.

[29] Y. Utumi, 'On rings of which any one-sided quotient rings are two-sided', Proc. Amer. Math. Soc. 14 (1963), 141-147.

Department of Mathematics

University of Louisiana at Lafayette

Lafayette LA 70504-1010

United States of America

e-mail: gfb1127@usl.edu

Department of Mathematics

Busan National University

Busan 609-735

South Korea

e-mail: jkpark@hyowon.cc.pusan.ac.kr
Department of Mathematics

Kyung Hee University

Suwon 449-701

South Korea

e-mail: jykim@nms.kyunghee.ac.kr 